\title{
Modelling and System Identification of a Stiff Stay Wire Fence Machine
}

\author{
C.E. Hann, D. Aitchison, D. Kirk and E. Brouwers
}

Department of Mechanical Engineering, University of Canterbury,

Christchurch, New Zealand 


\section{Abstract}

This paper investigates a severe gear backlash problem encountered in a stiff stay machine that is capable of producing a 26 line fence up to 2.6 metres in height at a speed of 80 stays/minute. Related problems in the literature, typically concentrate on the effect of gear backlash on the ability to control a shaft. However, in this case, very good control of the reference speed of the shaft was maintained in spite of the gear backlash. The problem was that the commanded torques were excessively large and threatened to damage the gear box. This problem motivated a complete analysis of the systems dynamics including the development of a model to better understand the response and allow the identification of external loads on the system. It was found that the method of sensing the shaft position (resolvers) was a major factor as well as the upgrading of the motor which was over responding to disturbances in the shaft. The model was validated using several torque limiting experiments and gave accurate prediction of the machine's major dynamics. The simulation tool developed provides the basis to predict the effect of different loads, wire types and/or motors on the machine for future designs minimizing the amount of experimentation on the machine. 


\section{Introduction and Motivation}

A stiff stay machine has been constructed that is capable of producing a twenty six line fence up to 2.6 metres in height at a speed of up to eighty stays per minute or one stay every 0.75 seconds. The wire fence machine consists of line wire, stay wire and knot wire feed mechanisms. The line wire is pulled up vertically and the stay wire is pushed across horizontally. The knot wire is placed 45 degrees across the line and stay wire before being cut, followed by the formation of the knot. Figure 1 shows an overall picture of the machine.

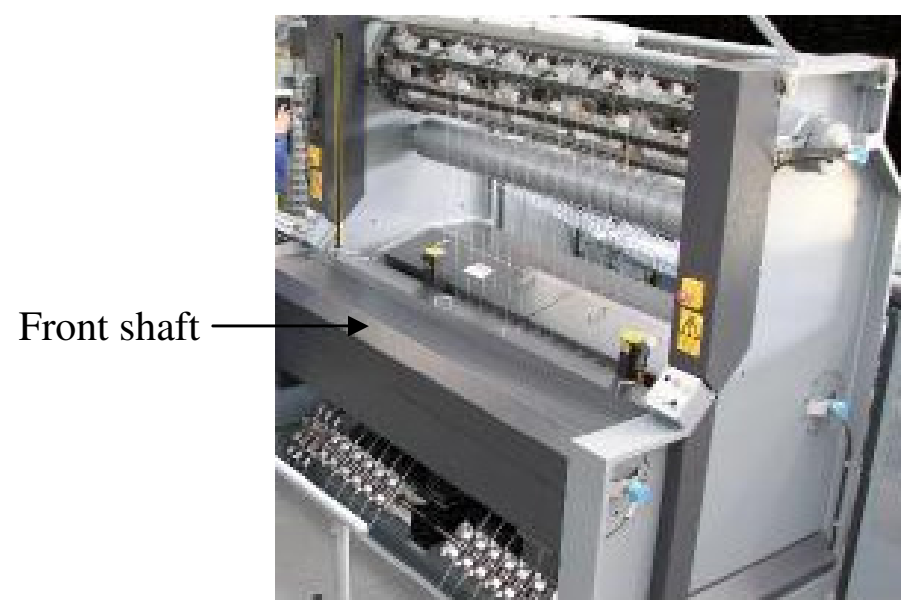

Figure 1 Picture of the stiff stay wire fence machine. The Front shaft is inside the casing denoted by an arrow.

The front shaft that is modelled in this paper has cams that drive the knot tying and cutting tools, so that a load goes onto the shaft whenever the knot is formed which occurs periodically. The majority of the load is taken by this front shaft when this knot is tied. Hence the shaft must be precisely controlled to deliver a reference angular position which is a function of time. The shaft is driven via a gearbox by an AC synchronous motor that provides a torque dependent on reference position data of the shaft defined so that the machine tools come together at precisely the same point in each knot tying cycle. The control system software/hardware of the motor is provided by Siemens and allows tight control of the shaft position.

There are many advanced feedback control systems that govern both the control of the front and back shafts, and provide safety mechanisms to avoid damage to the machine. The feedback shaft position data was initially provided by resolvers, and the control gains were obtained experimentally by trial and error on the machine. However, after upgrading the 
motor to $35 \mathrm{~kW}$, a problem arose with the drive gearbox. Specifically, very high motor torques and rates of torque were being commanded by the control system which resulted in significant gear backlash when the machine was brought up to speed. The result was a noisy gearbox which if run for too long may have caused damage to the gearbox and motor.

Gear backlash is a common problem in position control of machine tools resulting in the development of many sophisticated control strategies [1]. However, the emphasis is usually placed on improving position control rather than the physical effects of gear backlash itself $[2,3]$. For the application presented in this paper, the backlash had very little effect on the position control of the shaft and thus the timing of the machine tools. Specifically, when the resolver was placed at the far end of the shaft, and used for feedback to control the motor, motor torques were on the order of $275 \mathrm{Nm}$, while shaft position was controlled within 0.1 degrees of the required position. On the other hand, when the resolver was shifted to the motor drive end of the front shaft for feedback control, the motor torques were approximately halved but with little change in the controlled shaft position. The major reason for the large motor torques in the case of feedback from the far end of the shaft, is likely due to torsional resonance, which would be expected to be a maximum at the furthest distance from the drive end of the front shaft.

Hence, the backlash problem addressed in this paper is not focussed on it's effect on the quality of position control, but the effect in the gearbox, which resulted in a loud audible noise and potential damage longer term. To reduce the noise, one thought was to come up with a mechanical solution. Gear dynamics have been well studied and characterized in the literature [4-6] and thus present one possible approach to understanding and correcting the gear backlash. For example gear reduction or helical shaped teeth are well known to reduce gear backlash [4].

However, any mechanical solution to the problem like gear reduction, would be expensive and time consuming and thus it was not investigated. The preferred option was to pursue the idea that the motor was over responding to disturbances in the shaft and the various loads from the knot tying tools. Therefore, this backlash problem provided the initial motivation to fully characterize the major dynamics of the machine. Specifically, the goals were to understand the control mechanisms involved in the high motor torques and in the response of 
the shaft to the various loads. To achieve these goals, a mathematical model was developed for the system.

A further motivation for this research was to create a modelling methodology that can be generalized to predict the effect of different loads, wire types and/or motors on the machine for future designs. Therefore, the mathematical modelling was focused on capturing the measured shaft position response and motor torque inputs to allow prediction of various control system strategies without requiring extensive experimentation on the machine.

\section{Methodology}

\subsection{Modelling concept and comparisons to the literature}

The motor used to control the front shaft of the stiff stay machine is a 1FT6 synchronous motor [7]. The mathematical modelling of an AC synchronous motor is challenging, requiring many parameters and experiments to characterize the dynamics [8-10] including finite element analyses to fully describe the electromagnetic field behaviour [11]. However, the control system for converting a commanded current into a motor torque is based on well developed and validated Siemens software and the 1FT6 motor has very small torque ripple and almost constant torque characteristic [7]. Hence, it was decided to not model the AC motor. Instead, it was assumed that any commanded current is precisely delivered to the motor.

The dynamics of the interactions between geared shafts are well known and many models of varying complexity exist in the literature (e.g. [12-14]). For the application presented in this paper, the most important variable to be predicted is the maximum deviation of the shaft position away from the reference shaft position. This deviation needs to be within certain bounds to ensure that the knot tying tools do not crash together and cause significant and expensive damage to the system. Therefore, the mathematical model only needs to predict the maximum shaft position deviation response to different input parameters within these predefined safety limits. In other words, the complex time varying shaft dynamics and interactions with the applied load that correspond to shaft position deviations within the limits are not required to be modelled. 
Hence, the mathematical modelling methodology presented, is a minimal modelling approach where only the essential dynamics related to the practical engineering outcome are captured. The concept is to add further complexity as required to improve practical utility in the real system. The model and methods also need to be readily generalizable to other systems and parameters for example, different wire types, motors and knot tying procedures.

Note that different wire types and knot tying procedures will exert different external loads on the front shaft. Load torque is typically measured by a torque transducer (e.g. [15]), but direct measurement of the external torque applied to the front shaft in the stiff stay machine would be difficult due to the machine set up. Hence in this paper the external load is assumed to be an unknown parameter which must be identified from experiments using a model of the machine.

\subsection{Control system and mathematical model}

The behaviour of the front shaft in the wire fence machine is primarily governed by the control system and the resulting commanded motor torques that alter the main shaft dynamics as a function of the applied load. The control system for the front shaft consists of two main control loops, a position loop and a speed loop. These two loops have a major impact on the dynamics, hence the mathematical model is derived carefully with detailed discussion to ensure the control system configuration implemented on the machine is accurately represented.

The control system is designed to provide the appropriate motor torque to maintain a reference main shaft position that varies linearly as a function of time defined:

$$
R_{\theta}=v_{0} t
$$

where $v_{0}$ is the velocity in degrees/second, and corresponds to 360 degrees or 1 knot cycle for a given time period. The maximum speed of the machine is 80 stays per minute which is equivalent to $v_{0}=480$ degrees. Figure 2 shows a typical motor torque input and the corresponding shaft position angles $\theta=0,90,180$ and 360 degrees. The knot is tied between 
90 and 180 degrees corresponding to $t \simeq 0.18$ and $t \simeq 0.37$ seconds, which is when the greatest loads are on the shaft.

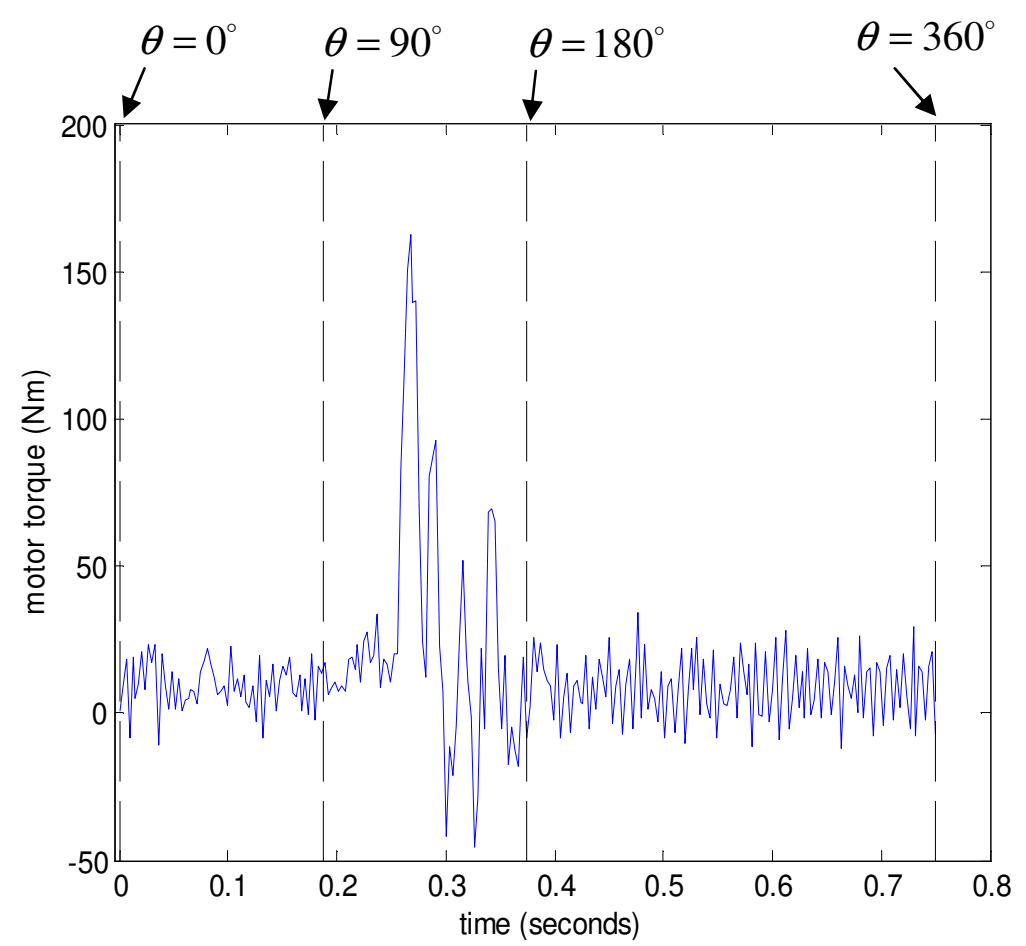

Figure 2 The input motor torque and shaft position in degrees. The greatest loads are when the shaft position angle is between 90 and 180 degrees.

Although it is the main shaft that is to be controlled, it is the motor shaft that provides the required torque. The main shaft and motor shaft are connected by a gearbox with a gear ratio defined:

$$
\text { gear ratio } \equiv k_{g}=26
$$

The mechanical dynamics of the main and motor shafts are well known and are described by the following differential equation:

$$
J_{\text {total }} \frac{d \omega}{d t}+b \omega=\tau_{\text {motor }}-\tau_{\text {ext }}
$$

where $J_{\text {total }}\left(\mathrm{Nm} \mathrm{s}^{2} / \mathrm{rad}\right)$ is the combined inertia and $b(\mathrm{Nm} \mathrm{s} / \mathrm{rad})$ is the combined damping for the main and motor shafts respectively, $\omega(\mathrm{rad} / \mathrm{s})$ is the motor shaft angular velocity, 
$\tau_{\text {motor }}(\mathrm{Nm})$ is the input motor torque and $\tau_{\text {ext }}(\mathrm{Nm})$ is the external torque from the applied load due to the knot tying procedure.

The reference speed $R_{\omega}(\mathrm{rad} / \mathrm{s})$ of the motor shaft is a proportional control dependent on the position of the shaft and is defined:

$$
\begin{aligned}
& R_{\omega}=\bar{K}_{1} \frac{\pi}{180}\left(R_{\theta}-\theta\right) \\
& \bar{K}_{1}=K_{1} \times k_{g}
\end{aligned}
$$

where $K_{1}(1 / \mathrm{s})$ is a proportional control chosen experimentally. For example, if the measured shaft position $\theta$ falls behind the reference position $R_{\theta}$, that is, $\theta<R_{\theta}$, the motor shaft needs to increase speed to catch up. This scenario is equivalent to an increase in the reference motor speed $R_{\omega}$. Similarly, if the shaft position gets in front of the reference position $R_{\theta}, \theta>R_{\theta}$, the motor shaft has to decrease speed.

To achieve the reference motor speed of Equation (4), a current is passed through the AC motor to produce the required torque via a proportional controller. The input current $i(\mathrm{~A})$ is defined:

$$
i=K_{2}\left(R_{\omega}-\omega\right)
$$

where $K_{2}(\mathrm{~A} \mathrm{~s} / \mathrm{rad})$ is the proportional gain found experimentally, $\omega(\mathrm{rad} / \mathrm{s})$ is the actual motor shaft speed and $R_{\omega}$ is the reference motor shaft speed given in Equation (3). The input motor torque $\tau_{\text {motor }}(\mathrm{Nm})$ in Equation (4), is proportional to the input current and is defined:

$$
\begin{aligned}
\tau_{\text {motor }} & =K_{T} i=K_{2} K_{T}\left(R_{\omega}-\omega\right) \\
K_{T} & =1.68 \mathrm{Nm} / \mathrm{A} \equiv \text { torque constant }
\end{aligned}
$$

The torque constant $K_{T}$ in Equation (8) is taken from the configuration manual for a 1FT6 synchronous motor. In practice the desired input current of Equation (5) is delivered by a PID control of the AC motor. However, as was discussed earlier, modelling of the AC motor 
is not required as it can be assumed that the desired current given by Equation (5) is precisely delivered to the motor.

The motor and main shaft dynamics of Equation (3) can be represented by the open loop transfer function:

$$
\begin{gathered}
G_{\text {motor }}(s)=\frac{\omega(s)}{\tau_{\text {net }}(s)}=\frac{1}{J s+b} \\
\tau_{\text {net }}=\tau_{\text {motor }}-\tau_{\text {ext }}
\end{gathered}
$$

where $\tau_{\text {net }}(\mathrm{Nm})$ is the torque due to the external load on the system. Using Equation (8), Equation (3) can be represented graphically as shown in Figure 3.

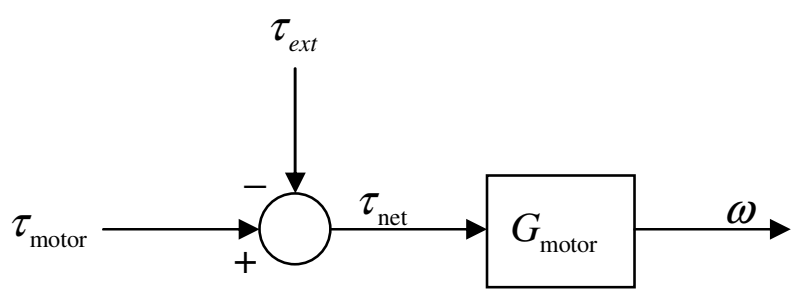

Figure 3 Graphical representation of the motor and main shaft dynamics of Equation (3)

Combining Equations (1)-(10) and utilizing the graphical terminology of Figure 3, an overall closed loop system model for the wire fence machine can be developed and is summarized in Figure 4. Note that the integrator $\int_{0}^{t}$ in Figure 4 refers to integrating the shaft velocity $\omega$ which is then multiplied by $\frac{180}{\pi} \cdot \frac{1}{k_{g}}$ to convert from the motor shaft position in radians to the main shaft position in degrees. The fedback $\omega$ in the speed loop is obtained by differentiating the motor shaft position angle $\theta_{\text {motor }}$. The angle $\theta$ is also directly fedback in the position loop as shown in Figure 4. In practice, the main shaft angle $\theta$ and motor shaft angle $\theta_{\text {motor }}$ are measured by separate resolvers or encoders. 


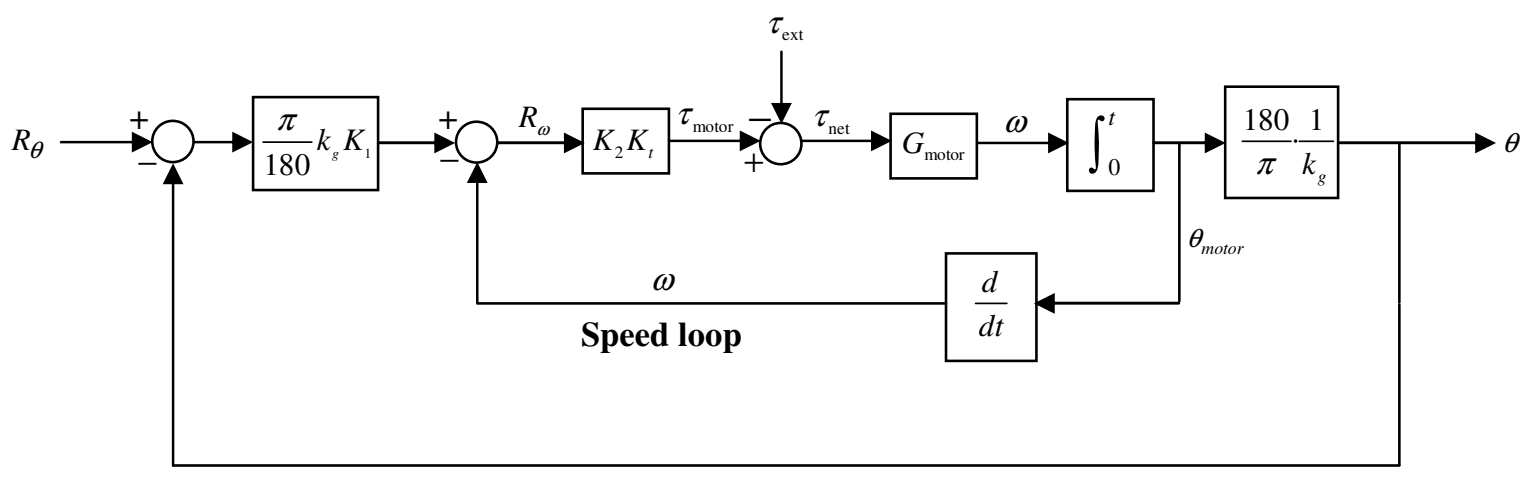

Position loop

Alternatively, Figure 4 can be written as a system of differential equations defined:

$$
\begin{aligned}
J \frac{d \omega}{d t}+b \omega & =K_{2} K_{t}\left(\frac{\pi}{180} k_{g} K_{1}\left(v_{0} t-\theta\right)-\omega\right)-\tau_{\text {ext }} \\
\frac{d \theta}{d t} & =\frac{180}{\pi} \cdot \frac{1}{k_{g}} \omega
\end{aligned}
$$

where $v_{0}$ is typically set at the maximum speed of $v_{0}=480 \% \mathrm{~s}$.

\subsection{Identifying load and system parameters - single line fence}

The proportional gains $K_{1}$ and $K_{2}$ in Equations (5) and (7) were worked out experimentally on the wire fence machine and are defined:

$$
K_{1}=258(1 / \mathrm{s}), \quad K_{2}=30(\mathrm{~A} \mathrm{~s} / \mathrm{rad})
$$

Note that taking into account the torque constant $K_{T}$ in equations (7) and (8), the effective gain $K_{2} K_{T}=50 \mathrm{Nm} \mathrm{s} / \mathrm{rad}$, which is the units implemented on the machine software. The unknown parameters in the system of Figure 4 are given by:

$$
\text { unknown parameters } \equiv\left\{\tau_{\text {ext }}(\mathrm{Nm}), b(\mathrm{Nm} \mathrm{s} / \mathrm{rad}), J\left(\mathrm{Nm} \mathrm{s}^{2} / \mathrm{rad}\right)\right\}
$$


Note that the inertia in the motor shaft is known from the configuration manual of the 1FT6 synchronous motor, but the inertia in the main shaft is unknown. Therefore since $J$ in Equation (12) is the combined inertia of the main and motor shafts, $J$ is also unknown. The measured known parameters of Figure 4 are:

$$
\text { known parameters } \equiv\left\{R_{\theta}, \theta, k_{g}, K_{1}, K_{2}, K_{t}, \tau_{\text {motor }}, \omega, R_{\omega}\right\} \text {, }
$$

Since $R_{\theta}$ is defined by the user, $\omega$ is the differential of $\theta$, and $R_{\omega}$ is determined by Equation (4), the two independent output parameters in Equation (13) that can be used to identify the parameters of Equation (12) are:

$$
\text { independent output parameters } \equiv\left\{\theta, \tau_{\text {motor }}\right\}
$$

The initial data set investigated was a single run of the machine on the single line stiff stay fence, with a speed of 70 stays per minute, or 1 stay in $\sim 0.86$ seconds. The angle $\theta$ in Equation (14) was measured by a resolver on the main shaft and the motor torque $\tau_{\text {motor }}$ was also measured. The damping $b$ in Equation (12) is estimated first by assuming that when $\theta \notin\left\{90^{\circ}, 180^{\circ}\right\}$, the external torque is 0 . In otherwords, during this period of no load, the motor torque $\tau_{\text {motor }}$ in Equation (3) is being applied only to overcome the damping in the combined main and motor shaft system. At steady state and with no load, there will be a constant torque $\tau_{\text {motor }}=\tau_{0}$, and hence constant shaft velocity $\omega=\omega_{0}$. Setting $\frac{d \omega}{d t}=0$ and solving for $\omega$ in Equation (3) yields:

$$
\begin{aligned}
b & =\frac{\tau_{0}}{\omega_{0}} \\
\tau_{0} & \equiv \text { constant torque with no load, } \\
\omega_{0} & \equiv \text { constant motor shaft velocity with no load }
\end{aligned}
$$

In practice $\tau_{0}$ and $\omega_{0}$ in Equation (15) can be estimated by the average of the measured motor torque and shaft velocity during the periods where $R_{\theta} \notin\left\{90^{\circ}, 180^{\circ}\right\}$. 
The aim of the model of Figure 4, is to predict the maximum deviation of the shaft position $\theta$ for various control strategies and changes in parameters. Therefore, a precise knowledge of the waveform shape of $\theta$ is not required, and hence a detailed model of $\tau_{\text {ext }}$ is not necessary. Given the relatively limited data available from this particular experiment, a simple model of $\tau_{\text {ext }}$ is defined:

$$
\begin{aligned}
\tau_{\text {ext }}(t) & =\alpha \tau_{\text {motor }}(t), R_{\theta}(t) \in\left[90^{\circ}, 180^{\circ}\right] \\
& =0, \text { otherwise }
\end{aligned}
$$

where $\alpha$ is an unknown parameter to be identified. The optimization of $\tau_{\text {ext }}$ and $J$ of Equation (12) is then reduced to the identification of $J$ and $\alpha$. Define:

$$
\begin{gathered}
\left\{\theta_{1}, \ldots, \theta_{n}\right\} \equiv n \text { measured main shaft positions } \\
\left\{\tau_{\text {motor }, 1}, \ldots, \tau_{\text {motor }, n}\right\} \equiv n \text { measured motor torques } \\
\left\{\omega_{1}, \ldots, \omega_{n}\right\} \equiv \text { motor shaft velocities from differentiation of } \theta_{i} \\
\quad \Delta t=\frac{T}{n} \equiv \text { sampling period of } \theta \text { and } \tau_{\text {motor }}, T=\text { period of machine }
\end{gathered}
$$

The parameters $\tau_{0}$ and $\omega_{0}$ that determine $b$ in Equation (13) are thus determined:

$$
\begin{aligned}
& \tau_{0}=\text { mean }\left\{\tau_{\text {motor }, i} \mid \theta_{i} \notin\left\{90^{\circ}, 180^{\circ}\right\}\right\} \\
& \omega_{0}=\operatorname{mean}\left\{\omega_{i} \mid \theta_{i} \notin\left\{90^{\circ}, 180^{\circ}\right\}\right\}
\end{aligned}
$$

For a given $\alpha$ from Equation (16), $b$ from Equations (15), (18) and (19), and $J$, the model of Figure 4 can be readily solved numerically in Simulink or Matlab. Define:

$$
\begin{aligned}
\left\{\hat{\theta}_{1}, \ldots, \hat{\theta}_{n}\right\} & \equiv \text { simulated outputs of } \theta \text { from Figure } 4 \\
\left\{\hat{\tau}_{\text {motor }, 1}, \ldots, \hat{\tau}_{\text {motor }, n}\right\} & \equiv \text { simulated outputs of } \tau_{\text {motor }} \text { from Figure } 4 \\
\text { sampling period } & \equiv \Delta t \text { from Equation }(17)
\end{aligned}
$$


Define:

$$
F(\alpha, J)=\frac{\left|\max _{i \in\{1, \ldots, n\}} \hat{\theta}_{i}-\max _{i \in\{1, \ldots, n\}} \theta_{i}\right|}{\left(\max _{i \in\{1, \ldots, n\}} \Delta \theta_{i}-\min _{i \in\{1, \ldots, n\}} \Delta \theta_{i}\right)}+\frac{\sqrt{\sum_{i=1}^{n} \frac{\left(\hat{\tau}_{\text {motor }, i}-\tau_{\text {motor }, i}\right)^{2}}{n}}}{\max _{i \in\{1, \ldots, n\}} \tau_{\text {motor }, i}}
$$

where:

$$
\Delta \hat{\theta}_{i}=R_{\hat{\theta}_{i}}-\hat{\theta}_{i}, \Delta \theta_{i}=R_{\theta_{i}}-\theta_{i}
$$

The parameters of Equation (22) are defined for convenience in plotting, since the angles of $\hat{\theta}$ and $\theta$ are not constrained to lie in $\left[0,360^{\circ}\right]$, and thus gradually increase over time. The angles and torques are non-dimensionalized to ensure approximately equal weighting between the two measurements. The optimum set of parameters $\alpha$ and $J$ are defined such that the objective function of Equation (21). This problem can be readily solved in Matlab using standard non-linear regression. The overall procedure is summarized in Figure 5.

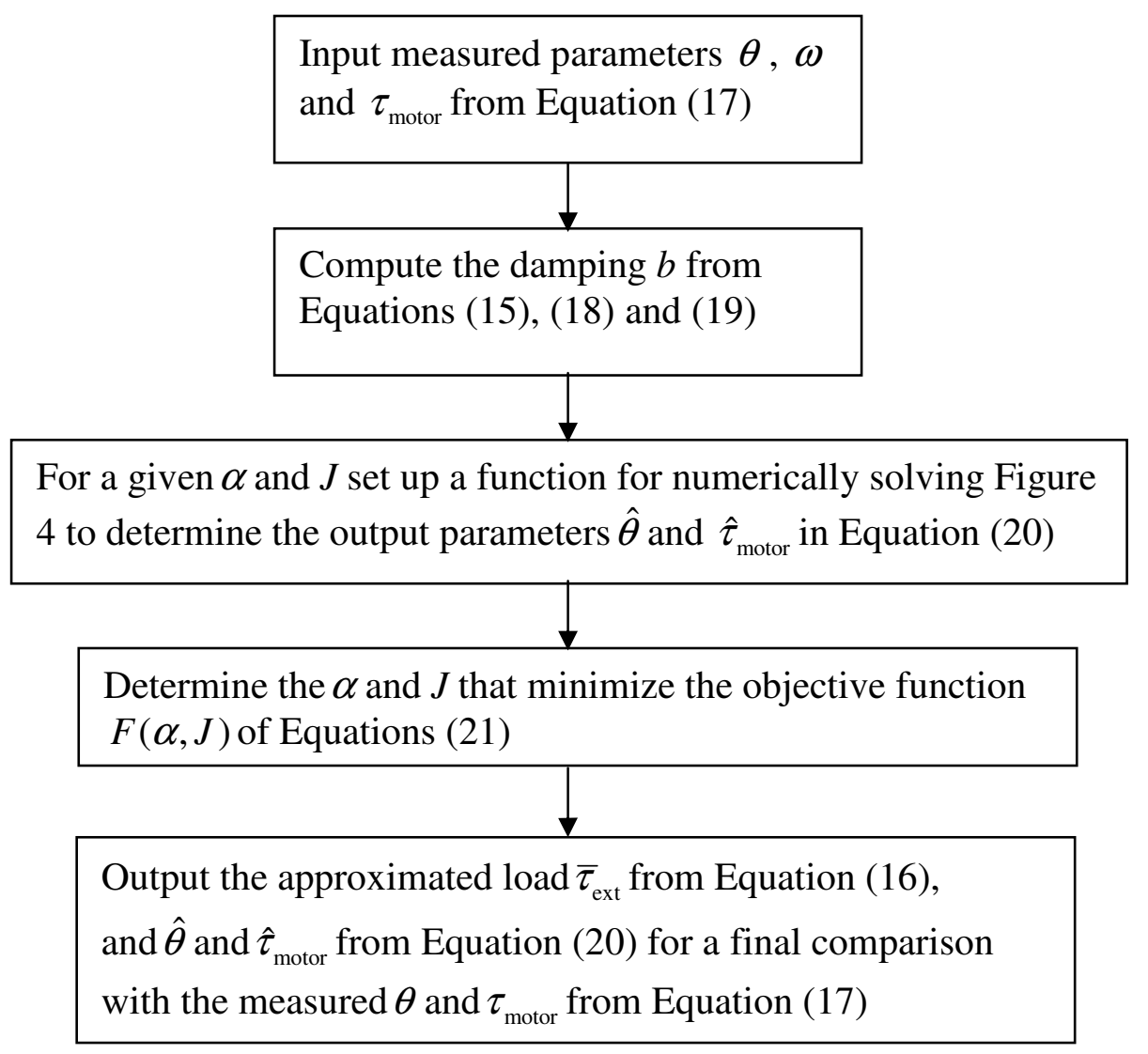

Figure 5 Algorithm for determining the unknown parameters $b, J$ and $\tau_{\text {ext }}$ 


\subsection{Identifying load and system parameters - dual line fence}

A dual line fence creates a slightly greater load than a single line fence on the main and motor shafts, but the model of Figure 4 is equally valid in this case. Several torque limiting experiments were applied on the machine. Specifically, the maximum input motor torque was changed incrementally from no limit, 130, 110, 100 and $80 \mathrm{Nm}$ respectively. Furthermore, for the case of $80 \mathrm{Nm}$, the minimum input torque was set to $-30 \mathrm{Nm}$ followed by $0 \mathrm{Nm}$, which gave a total of 6 separate runs on the machine. The number of stays per minute was set at the maximum allowable of 80 , which is equivalent to 1 stay every 0.75 seconds.

The 6 experiments give significant data to validate the model of Figure 4 and to allow a characterization on what motor torque requirements are needed to adequately control the main shaft. In addition, the more severe torque limiting values of $110 \mathrm{Nm}$ and below effectively bypass the control system during the periods of high load. Therefore, the effect of the load on the shaft is separated from the control system, simplifying the identification of the load and improving accuracy in the model. Comparisons with the case of no torque limit, can be made to better understand the effectiveness of the control system and any potential interaction with the load. A systematic method for identifying different time varying loads on the machine which would change for different wire types may lead to design improvements in the future.

The method for identifying the system parameters of Equation (12) is similar to the algorithm of Figure 5, but the wealth of data available means a more accurate model of the load can be considered. Let $\hat{t}_{1}, \ldots, \hat{t}_{10}$ be 10 time points spaced around the period of maximum load, which is approximated using the measured motor torque. The specific points are chosen based on satisfactorily capturing the motor torque response with no torque limit, with a piecewise linear approximation. Thus an estimate of the resolution needed to represent the time varying behaviour of the load is obtained. Outside this maximum load period, the external torque is assumed to be 0 , to minimize the number of parameters in the resulting optimization. The model for the external torque is defined: 


$$
\begin{aligned}
\tau_{\text {ext }} & =\tau_{i}+\frac{\left(\tau_{i+1}-\tau_{i}\right)}{\left(\hat{t}_{i+1}-\hat{t}_{i}\right)}\left(t-\hat{t}_{i}\right), t \in\left[\hat{t}_{i}, \hat{t}_{i+1}\right], i=1, \ldots, 9 \\
& =0, t \notin\left[\hat{t}_{1}, \hat{t}_{10}\right]
\end{aligned}
$$

An example profile is shown in Figure 6.

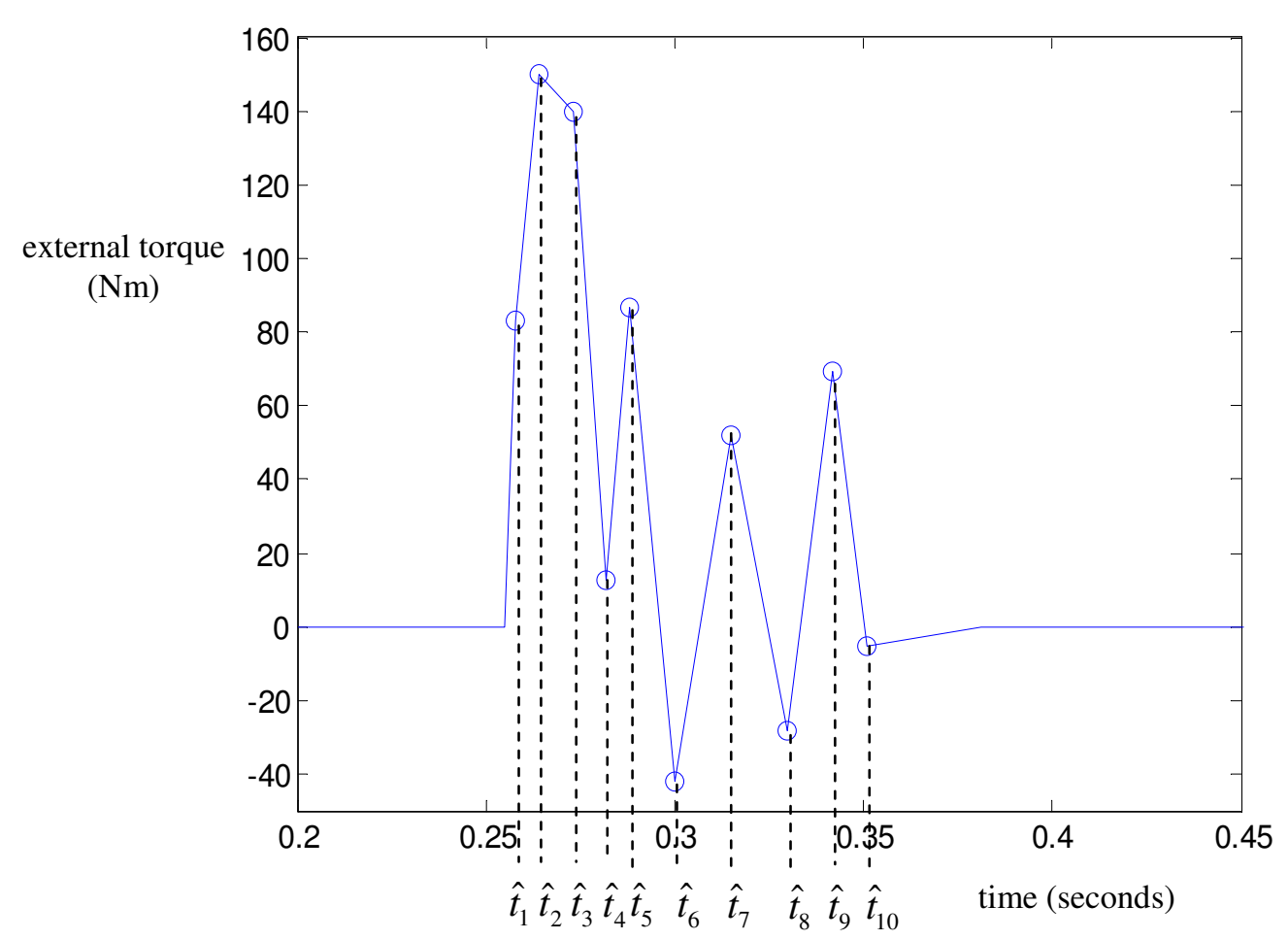

Figure 6 An example profile of the external torque in Equation (23)

There are 10 unknown parameters $\tau_{1}, \ldots, \tau_{10}$ in Equation (23) which combined with the parameters $J$ and $b$ gives 12 values to be optimized:

$$
\text { unknown parameters } \equiv\left\{\tau_{1}, \ldots, \tau_{10}, b, J\right\}
$$

The measured data for identifying the parameters in Equation (24) consists of up to 6 data sets. Superscripts (1),..,(6) are used to denote each of the datasets:
(1) $\equiv$ no torque limits
(2) $\equiv 130$ upper torque limit
(3) $\equiv 110$ upper torque limit 
(4) $\equiv 100$ upper torque limit

(5) $\equiv 80$ upper torque limit, -30 lower torque limit

(6) $\equiv 80$ upper torque limit, 0 lower torque limit

The measured data is defined:

$\left\{\theta_{1}^{(i)}, \ldots, \theta_{n}^{(i)}\right\} \equiv n$ measured main shaft positions for data set (i)

$\left\{\tau_{\text {motor },{ }^{(i)}}, \ldots, \tau_{\text {motor }, m}{ }^{(i)}\right\} \equiv m$ measured motor torques for data set (i) during maximum load $\left\{\omega_{1}^{(i)}, \ldots, \omega_{n}^{(i)}\right\} \equiv$ motor shaft velocities from differentiation of $\theta_{i}$ sampling period $\equiv \Delta t$ from Equation (17) $i \in\{1, \ldots, 6\}$

Similar to Equation (20), the simulated data outputs from the model of Figure 4, that are compared with the measured data in Equation (26), are defined:

$$
\begin{gathered}
\left\{\hat{\theta}_{1}^{(i)}, \ldots, \hat{\theta}_{n}^{(i)}\right\} \equiv \text { simulated outputs of } \theta \text { from Figure } 4, \text { for data set (i) } \\
\left\{\hat{\tau}_{\text {motor, } 1}^{(i)}, \ldots, \hat{\tau}_{\text {motor }, n}{ }^{(i)}\right\} \\
\text { sampling period } \equiv \Delta t \text { from Equation }(17), \quad i \in\{1, \ldots, 6\}
\end{gathered}
$$

Note that to obtain the simulated data in Equation (27), the model of Figure 4 is simulated numerically in Matlab for each torque limiting scenario in Equation (25).

Mathematically, upper and lower bounds UB and LB of the motor torque are implemented by redefining Equation (10):

$$
\begin{aligned}
J \frac{d \omega}{d t}+b \omega & =\hat{\tau}_{\text {motor }}-\tau_{\text {ext }} \\
\hat{\tau}_{\text {motor }} & =\max \left\{\min \left\{\tau_{\text {motor }}, \mathrm{UB}\right\}, \mathrm{LB}\right\} \\
\tau_{\text {motor }} & =K_{2} K_{t}\left(\frac{\pi}{180} k_{g} K_{1}(480 t-\theta)-\omega\right)
\end{aligned}
$$

An objective function is defined in a similar way to Equation (21): 


$$
F\left(\tau_{1}, \ldots, \tau_{10}, J\right)=\frac{1}{12} \sum_{j=1}^{6} \sqrt{\left(\max _{i \in[1, \ldots, n\}} \hat{\theta}_{i}^{(j)}-\max _{i \in\{1, \ldots, n\}} \theta_{i}^{(j)}\right)^{2}+\left(\min _{i \in\{1, \ldots, n\}} \hat{\theta}_{i}^{(j)}-\min _{i \in\{1, \ldots, n\}} \theta_{i}^{(j)}\right)^{2}}
$$

However, no motor torque data is used in this case since there is sufficient position data available from the 6 experiments. In addition, the resulting modified motor torque can then be compared to the measured motor torque to provide further model validation.

The optimal $\tau_{1}, \ldots, \tau_{10}$ and $J$ are defined such that $F$ in Equation (29) is minimized. Once $\tau_{1}, \ldots, \tau_{10}$ are known the external load is given by Equation (23). The overall algorithm is summarized in Figure 7.

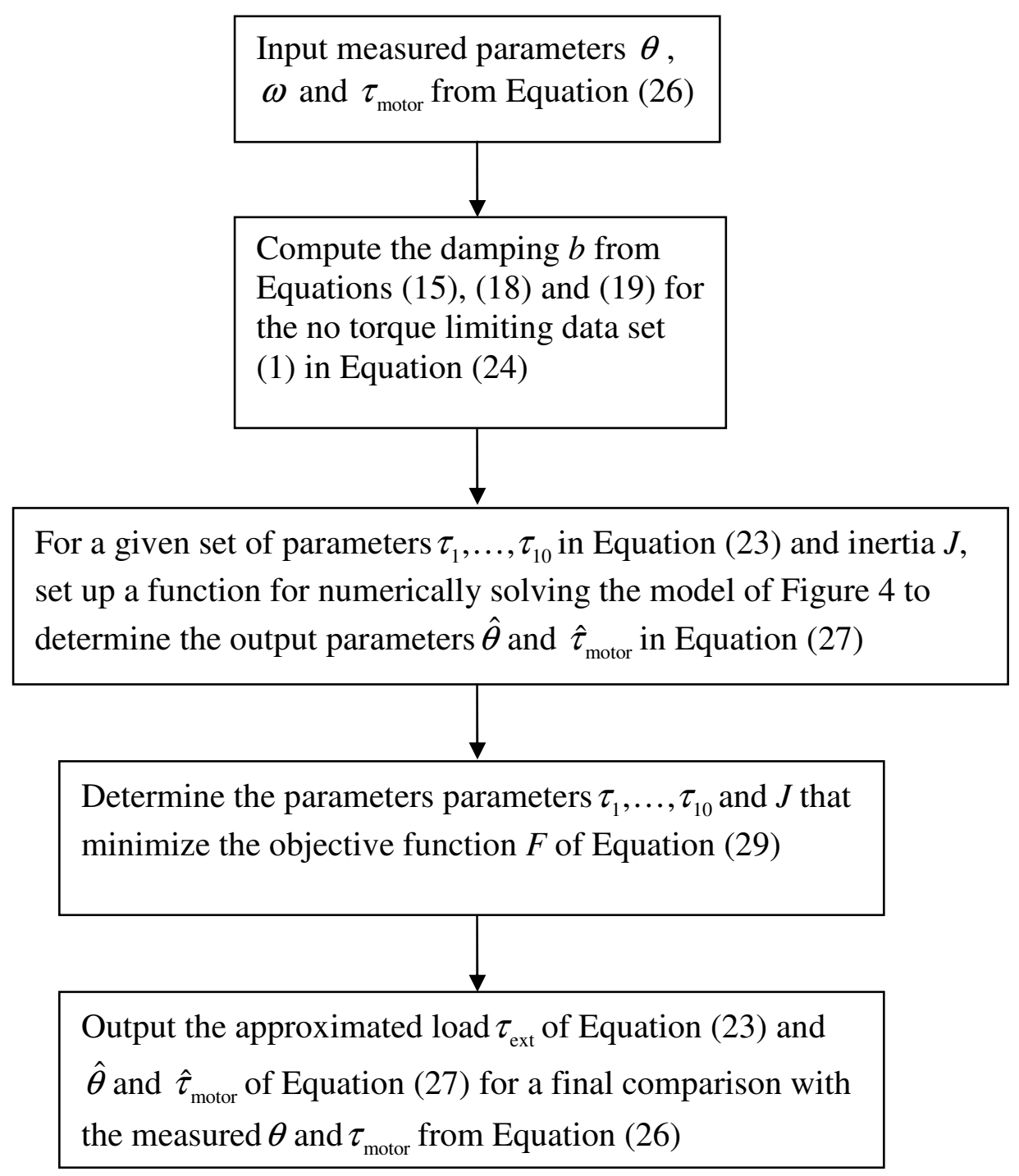

Figure 7 Algorithm for determining the unknown parameters $b, J$ and $\tau_{\text {ext }}$ 


\section{Results and Discussion}

\subsection{Single line fence}

The initial data analysed was one run, with no torque limit at a speed of 70 stays per minute, or with $v_{0}=420$ degrees $/ \mathrm{s}$ in Equation (1). A resolver was used to measure the main shaft position. The algorithm of Figure 5 is applied to identify the external load $\tau_{\text {ext }}$ using the approximate model of Equation (16), and the damping and inertia $\mathbf{J}$ from Equation (12).

The optimized parameters are defined:

$$
\text { Optimized parameters } \equiv\{b=0.0045, J=0.015, \alpha=0.85\}
$$

Substituting $\alpha=0.85$ into Equation (16) gives a maximum external load of $115 \mathrm{Nm}$. The model outputs are then compared to the measured position error and motor torque as shown in Figure 8.

(a)

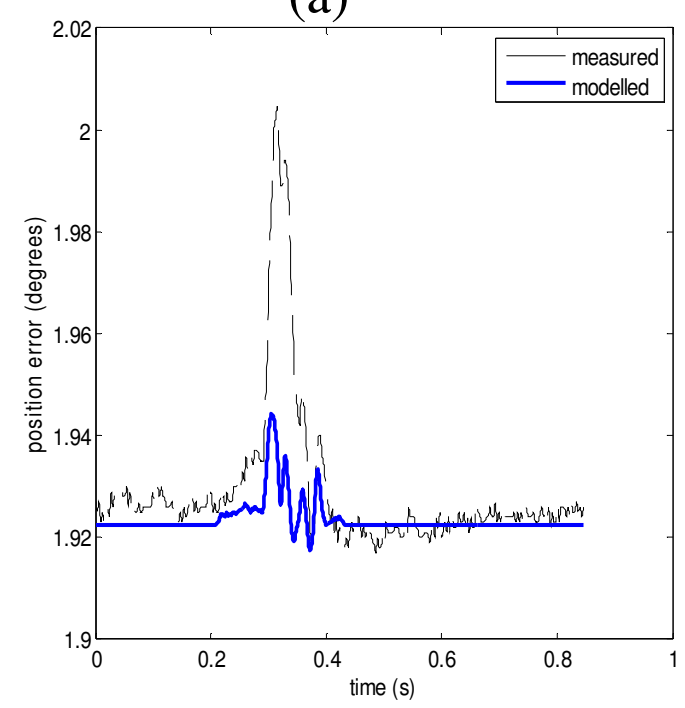

(b)

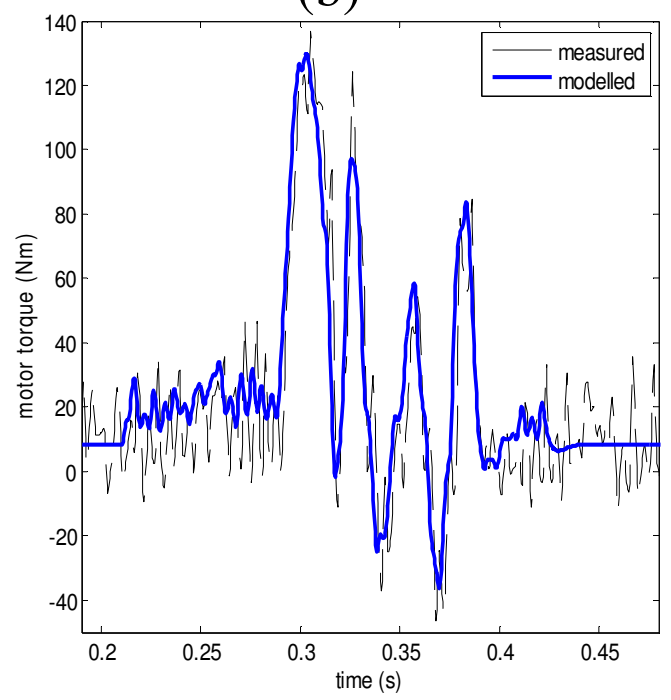

Figure 8 (a) Measured versus modeled position error. (b) Measured versus modeled motor torque

Overall, the modelled responses capture the main dynamics of the system including an accurate match to the steady state position error without load. The maximum position error is $\sim 0.06^{\circ}$ which corresponds to $3.1 \%$ of the offset position error of 1.92 degrees and is relatively small. The overall behaviour of the modelled motor torque closely represents the measured motor torque as shown in Figure 8 (b). 
Note that reducing the proportional gain $\mathrm{K}_{2}=30$ in Equation (11) to $14 \mathrm{~A}$ s/rad yields a more accurate match to the position error with minimal change in motor torque as shown in Figure 9.

(a)

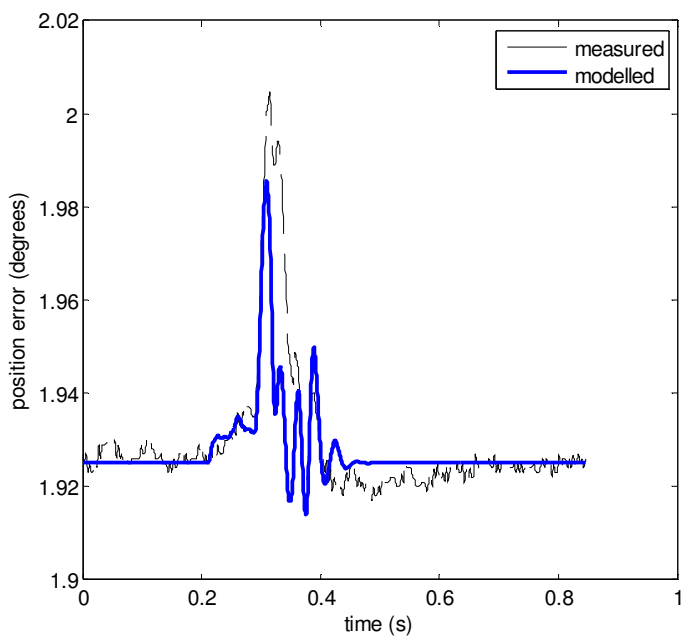

(b)

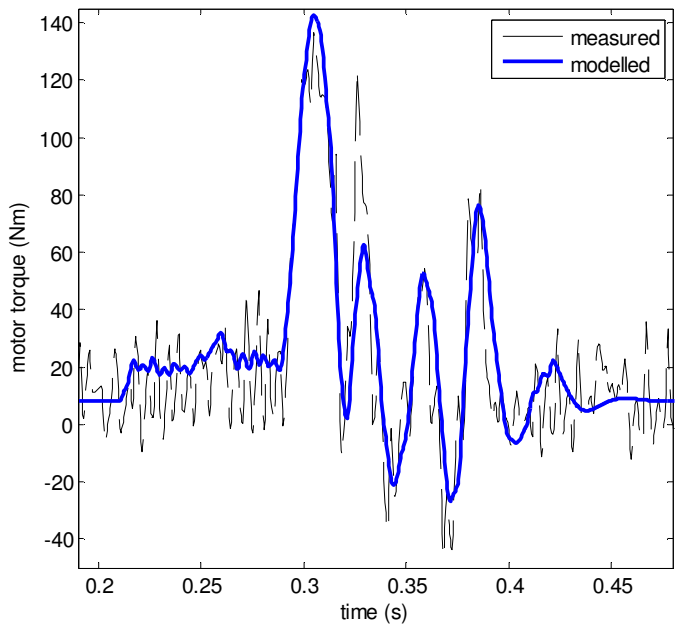

Figure 9 (a) Position error match with $\mathrm{K}_{2}=14$ in Equation (11) (b) motor torque match with $\mathrm{K}_{2}=14$

Figure 9 suggests that the speed loop control system of the machine is not performing as it should. This result may be due to interactions with torsional effects in the shaft and/or errors in the fedback position from the resolver. Torsional effects are known to occur in the shaft from vibrational tests performed at the two ends of the shaft. The vibration at the far end has displacement approximately twice that of the motor end. Furthermore, the commanded torques when the resolver is placed at the end of the shaft are 2-3 times greater than when the resolver is placed at the motor end. Thus, there are significant differences in the accelerations and displacements at both ends of the shaft. In addition to these effects, errors in a resolver are known to occur during high loads or accelerations which are present in this system, and resolvers are less accurate than encoders $[16,17]$.

To investigate the effect of increased error in the position measurement, noise is added to $\theta$ in the model of Figure 4 and a time delay is also considered. Figure 10(a) gives the position error from the model, and Figure 10(b) plots the net torque $\tau_{\text {net }}$ in Equation (10), which determines the net movement of the motor shaft. No noise or time delay is present in $\theta$ for this case of Figure 10. 
(a)

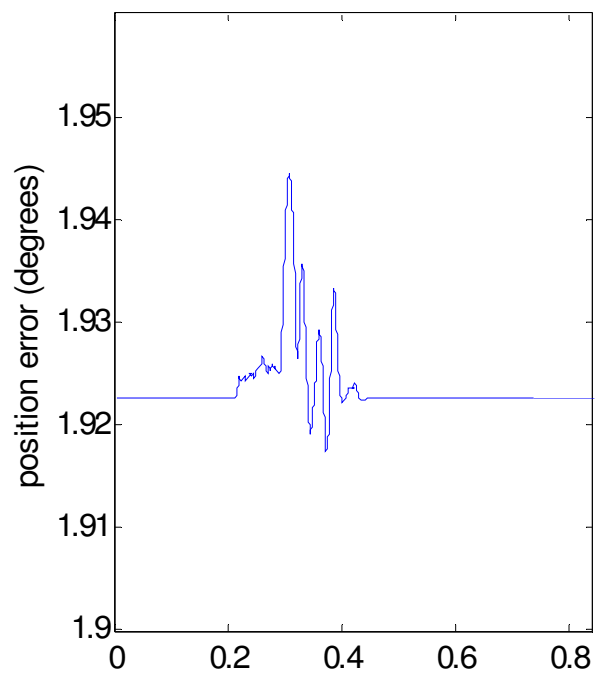

(b)

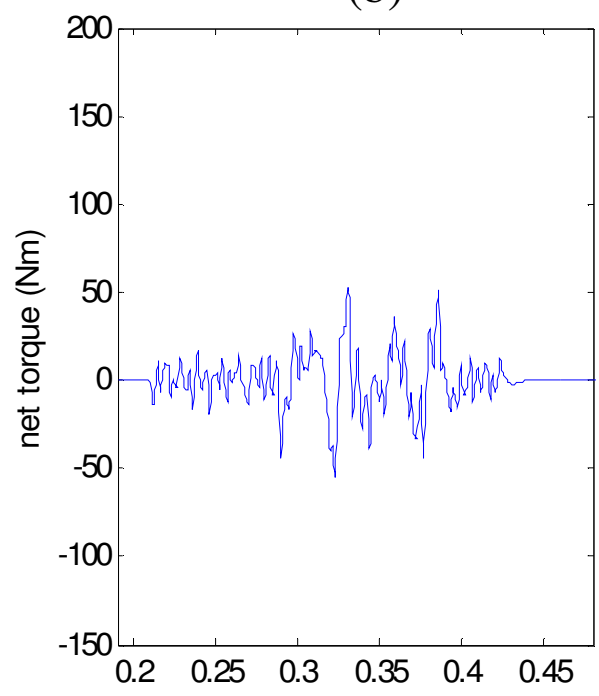

Figure 10 Modelled Position error and net torque with no noise

To represent noise, a median filter is applied to the measured position error of Figure 8 This median filter is then subtracted from the positional error to create the noise. Two orders of 8 and 10 are chosen for the medfilter to mimic an increasing amount of noise, Time delays of 0.0005 and 0.0001 seconds are also chosen. Figures 11 and 12 show the effect of increasing levels of noise on the position.

(a)

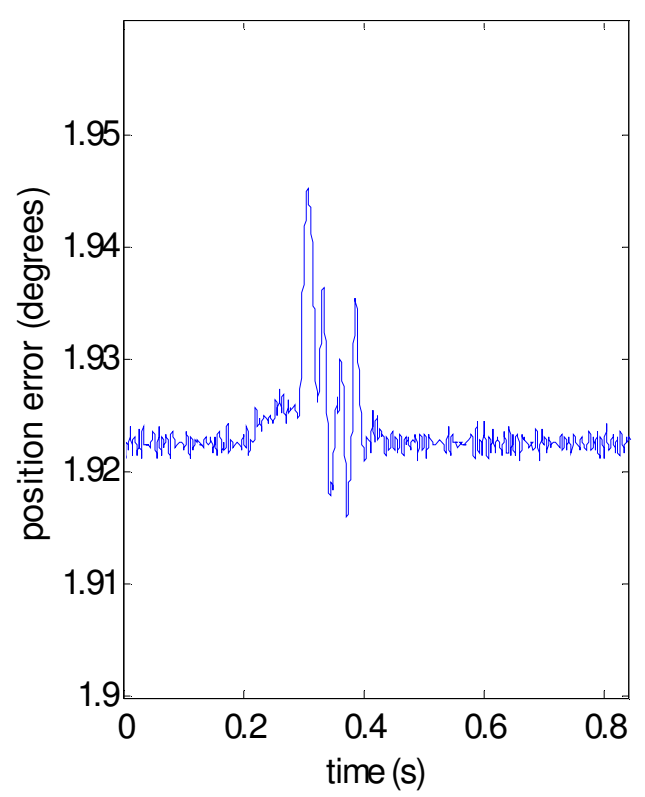

(b)

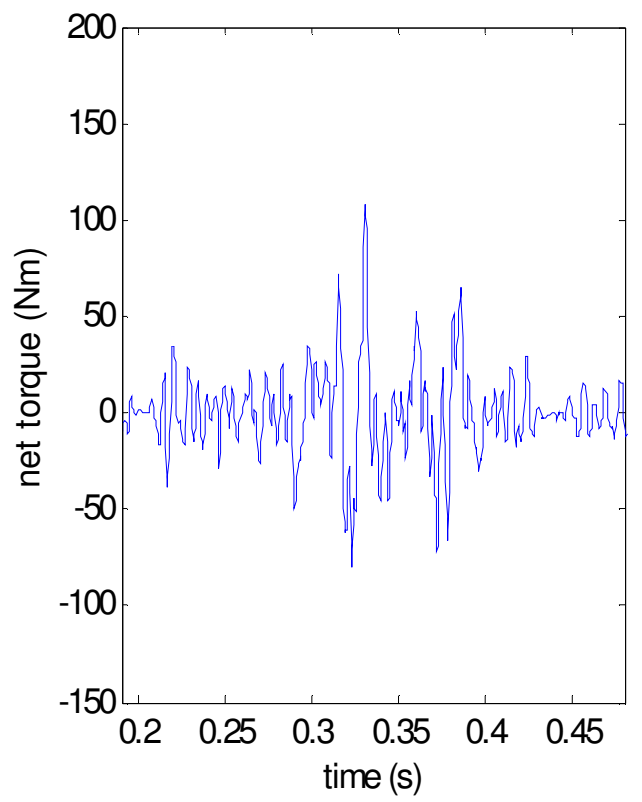

Figure 11 Position and net torque with noise created by a median filter of order 8 and time delay of 0.0005 seconds 
The controlled position for the shaft is not significantly affected by the noise as shown in Figures 11(a) and 12(a). However, the noise has a major effect on the net torque with an increase in the peak net torque from $50 \mathrm{Nm}$ to $150 \mathrm{Nm}$. The large increase and sensitivity of the net torque to errors in position suggest that inaccuracies in the resolver may be the reason for the loud audible noise heard in the gear box. Later experiments have showed that replacing the resolver by an encoder reduces this audible noise to as acceptable level confirming the trends observed in Figures 10-12.

(a)

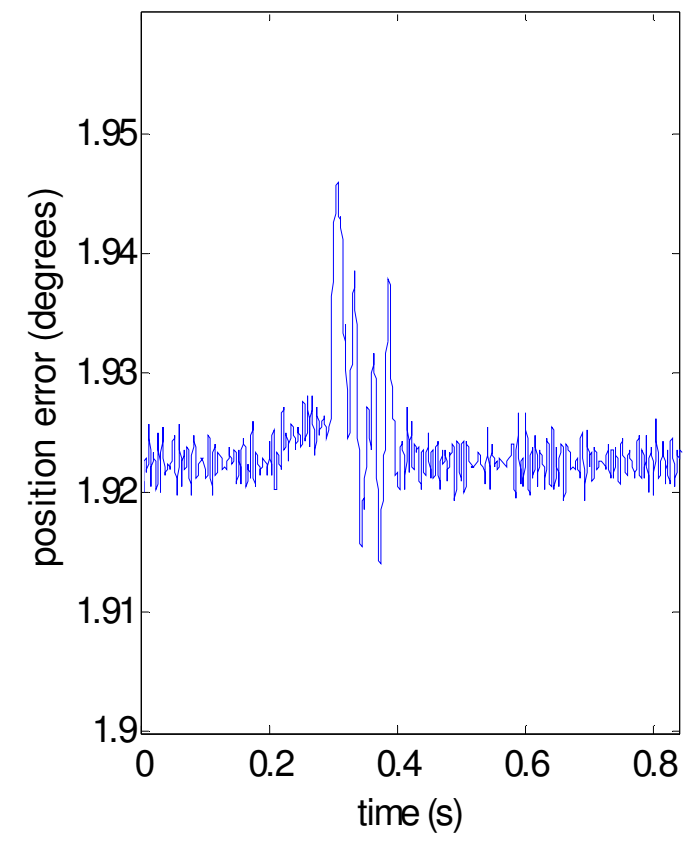

(b)

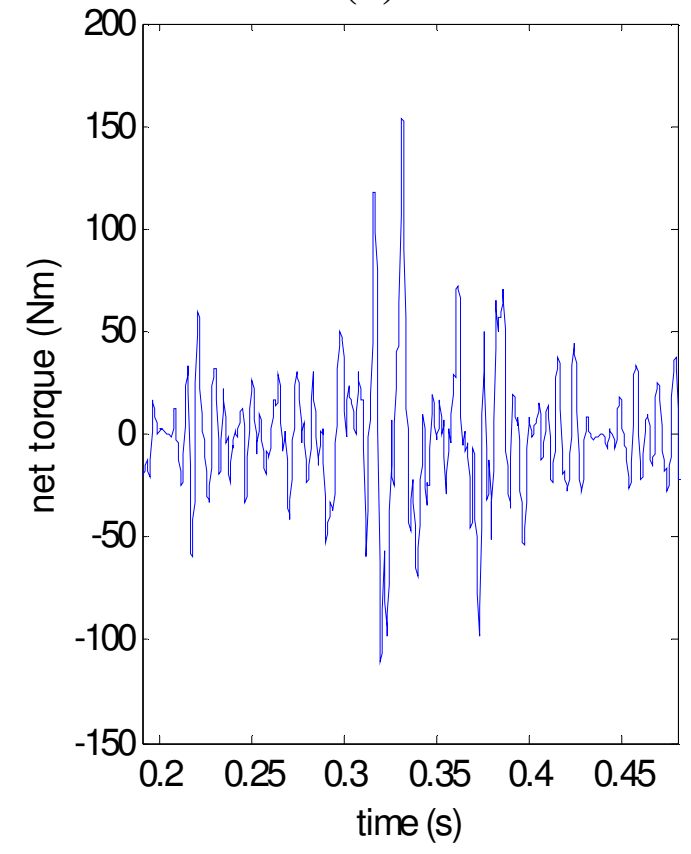

Figure 12 Position and net torque with noise created by a median filter of order 10 and time delay of 0.0001 seconds.

\subsection{Dual Line Fence}

The final data set analysed was 6 torque limiting experiments detailed in Equation (25). Again, a resolver was the means to measure the main shaft position. The machine was run at the maximum speed of 80 stays per minute, or equivalently $v_{0}=480$ degrees/s in Equation (1). The method of Figure 7 is applied to identify the external load parameters $\tau_{1}, \ldots, \tau_{10}$ in Equation (23), the inertia $\mathrm{J}$ and damping $\mathrm{b}$ in Equation (23). All the measured data of Equation (26) from the 6 experiments in Equation (25) is used. The optimized parameters are defined: 
Optimized parameters $\equiv\left\{b=0.02, J=0.03, \tau_{1}=44.9, \tau_{2}=147.7, \tau_{3}=102.7, \tau_{4}=-9.2\right.$

$$
\left.\tau_{5}=34.8, \tau_{6}=41.0, \tau_{7}=57.1, \tau_{8}=-37.9, \tau_{9}=61.0, \tau_{10}=-5.0\right\}
$$

and the identified external load $\tau_{\text {ext }}$ in Equation (23) is plotted in Figure 13.

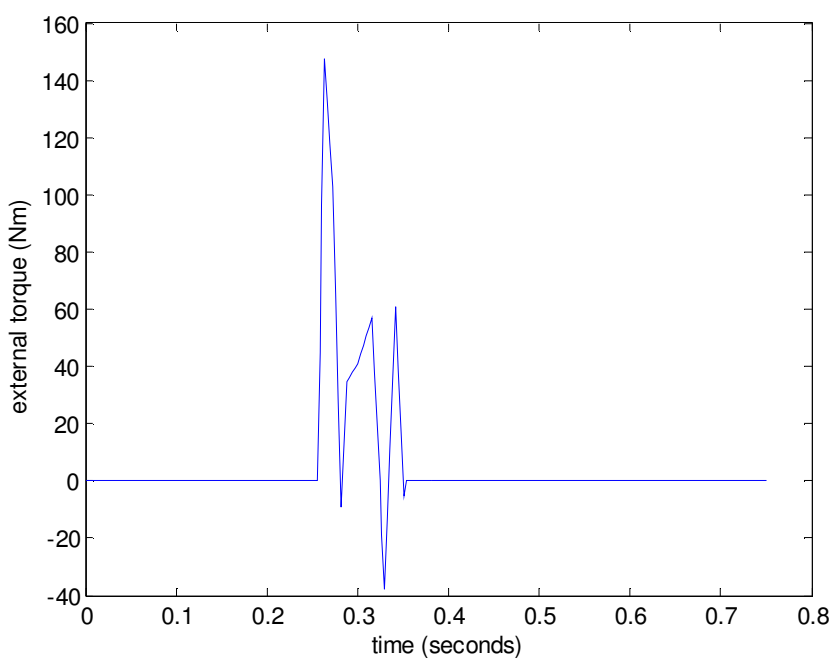

Figure 13 Identified external load of Equation (22)

The parameters of Equation (3) and $\mathrm{K}_{1}$ and $\mathrm{K}_{2}$ from Equation (11) are used to simulate the model of Figure 4 using the torque limit defined in Equation 3. The results are given in Figures 14-16 which show a good match to the position data, validating the model and methods. The motor torque data was not used to identify the parameters in Equation (31) but the overall motor torque response and trends are accurately captured further validating the approach. Specifically, the offset is accurately captured and there are good matches to both the maximum and minimum position errors. For example, the lower dip in Figure 16 for the data set with $\mathrm{UB}=80 \mathrm{Nm}$ and $\mathrm{LB}=0 \mathrm{Nm}$ is correctly predicted. Furthermore, in the model response the time spent at the maximum input motor torque increases as the upper bound (UB) is decreased. This behaviour matches a similar trend in the measured motor torque responses. Finally, the trend and magnitudes of the maximum position error as a fraction of the various torque limiting the scenarios is accurately represented by the model. This dynamic is the most important, as the maximum position error determines the safety in the machine, and this information can be used to ensure the machine tools are not damaged when various loads or controlled scenarios are applied. 

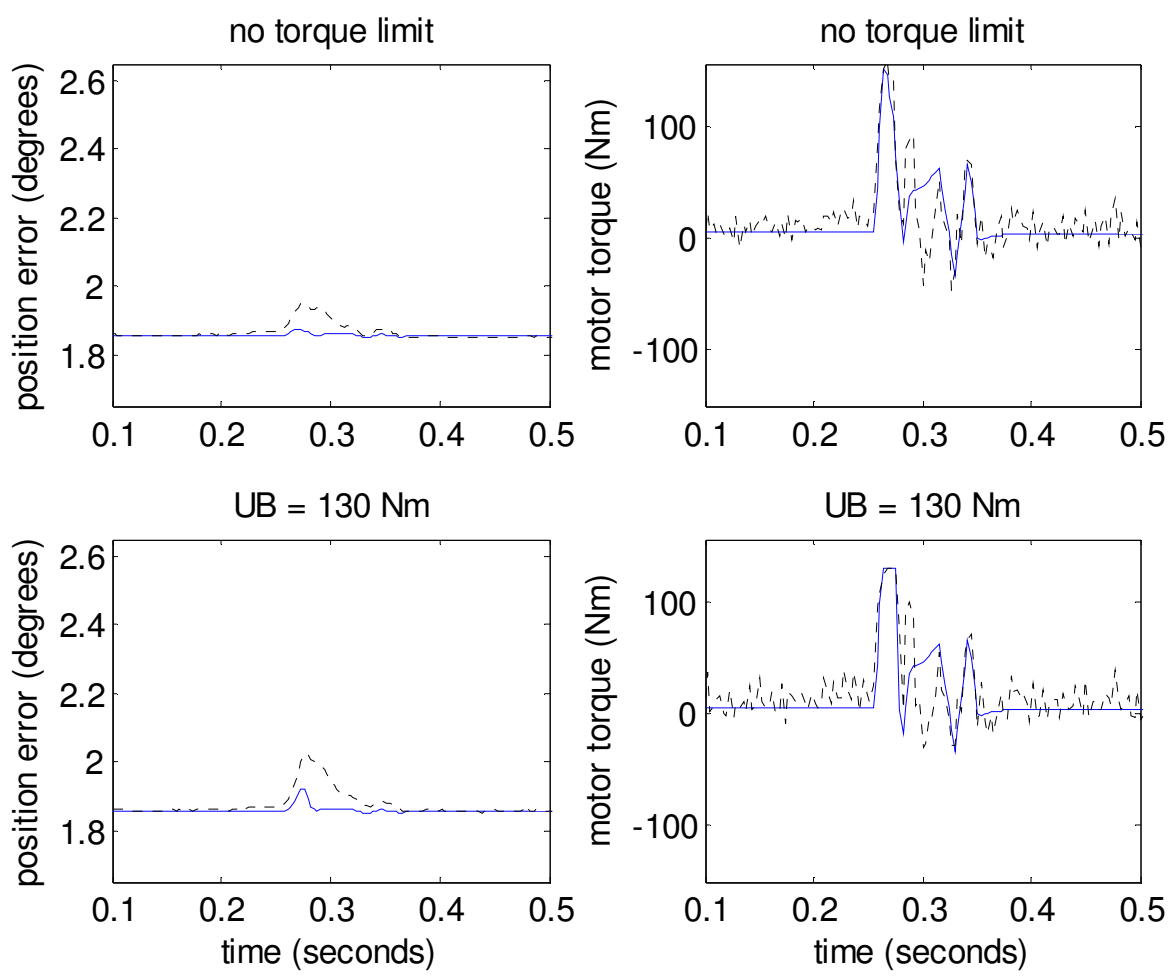

Figure 14 Modelled versus measure position error and motor torque for data sets (1) and (2) of Equation (25). The measured data is denoted by dashed lines and the modelled data is denoted by solid lines
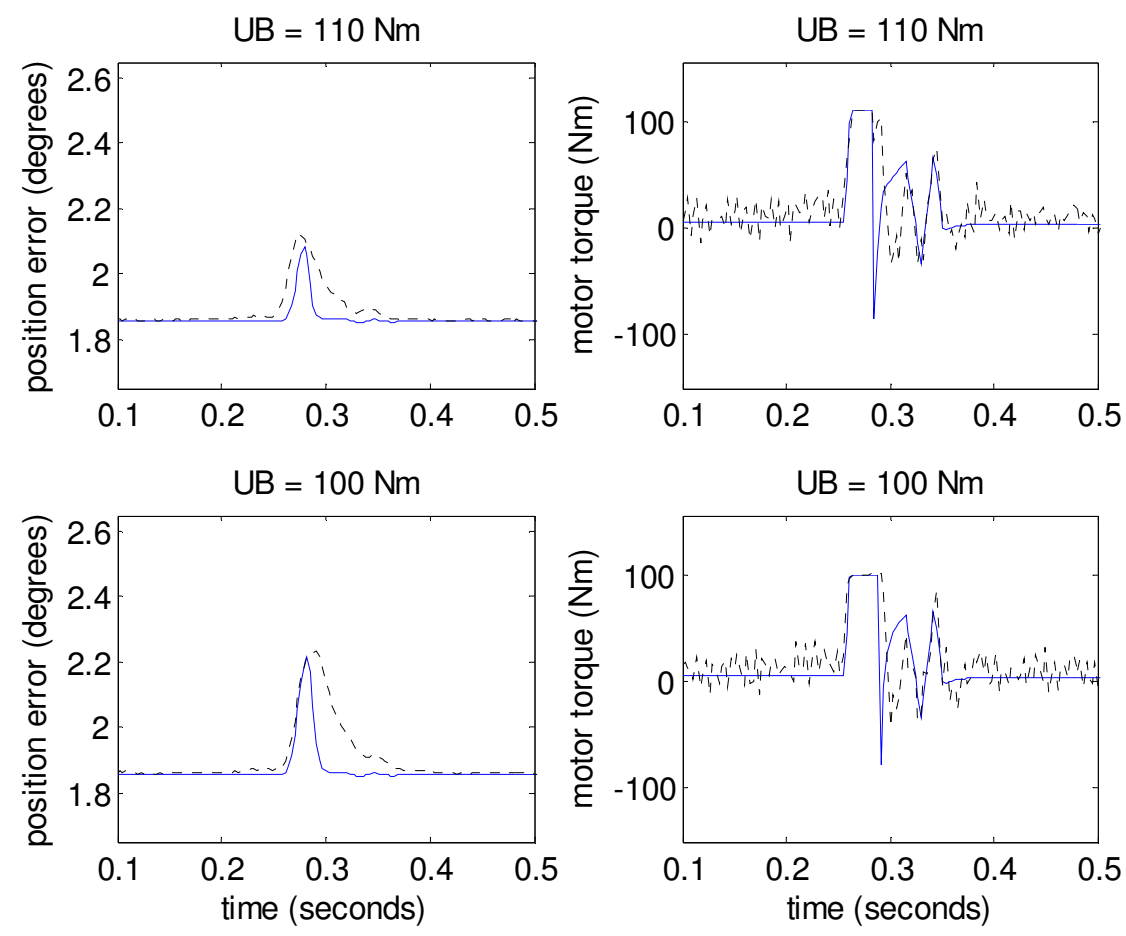

Figure 15 Modelled versus measure position error and motor torque, for data sets (3) and (4) of Equation (25). The measured data is denoted by dashed lines and the modelled data is denoted by solid lines. 

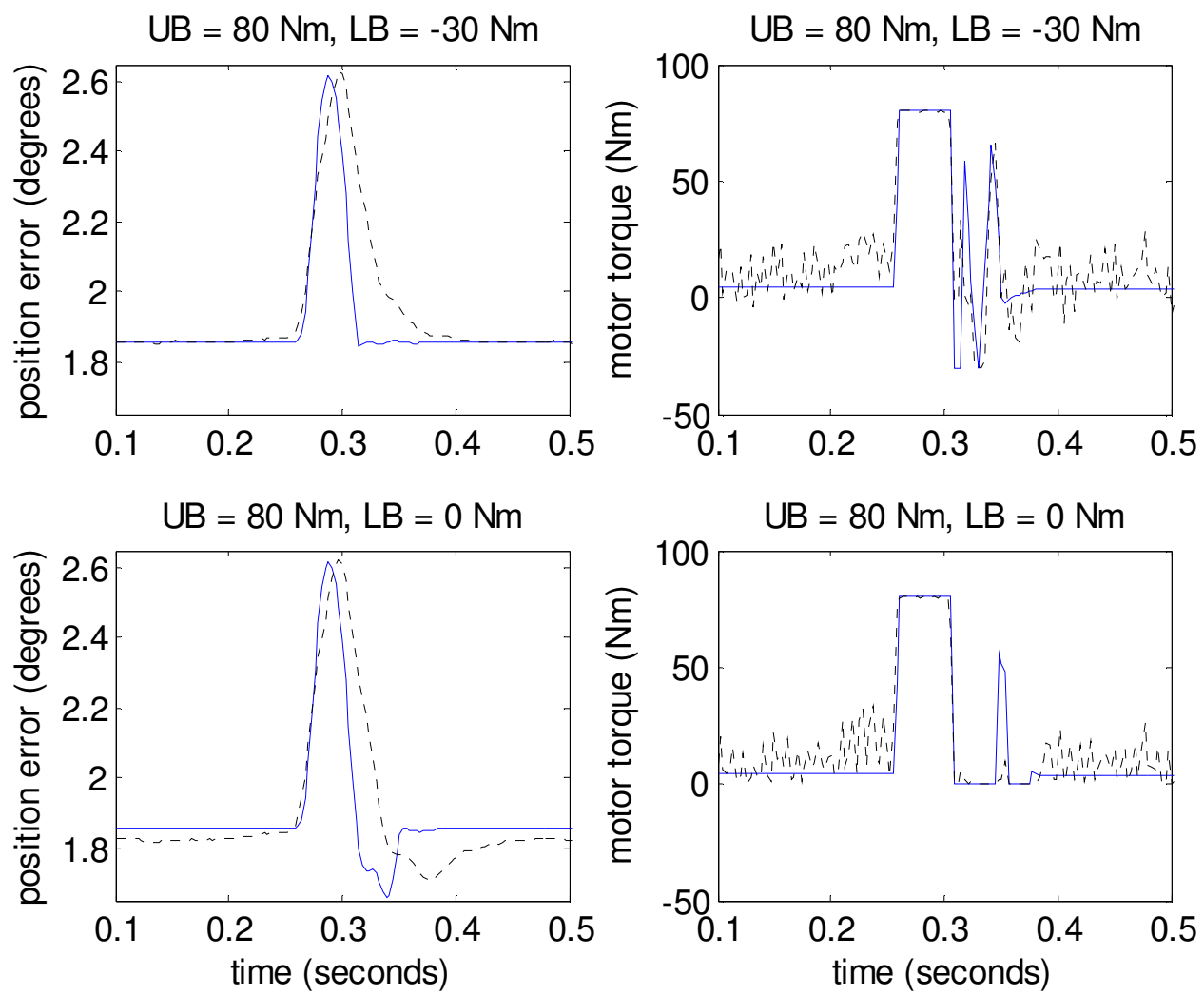

Figure 16: Modelled versus measure position error and motor torque for data sets (5) and (6) of Equation (25). The measured data is denoted by dashed lines and the modelled data is denoted by solid lines.

The differences between the modelled and measured maximum/minimum position errors in Figures 14-16 as defined in Equation (29) are shown in Table1. The parameters $\Delta \hat{\theta}$ and $\Delta \theta$ are defined in Equation (22).

Table 1: Model response position error differences for main shaft using all data sets (1)-(6) in Equation (25)

\begin{tabular}{|l|l|l|}
\hline Data Set & $\max \Delta \theta-\max \Delta \hat{\theta}$ & $\min \Delta \theta-\min \Delta \hat{\theta}$ \\
\hline$(1)$ & 0.074 & -0.001 \\
\hline$(2)$ & 0.074 & 0.004 \\
\hline$(3)$ & 0.038 & 0.006 \\
\hline$(4)$ & 0.018 & 0.005 \\
\hline$(5)$ & 0.015 & 0.007 \\
\hline$(6)$ & 0.009 & 0.049 \\
\hline
\end{tabular}


Note that the error in data sets (1) and (2) of Figure 14 can be significantly reduced by decreasing the proportional gain $\mathrm{K}_{2}$ in Equation (6) to $8 \mathrm{~A} \mathrm{~s} / \mathrm{rad}$. The result after this reduction of $\mathrm{K}_{2}$ is given in Figure 17, which shows a better match to the measured position error than Figure 14, with a minimal change in the motor torque. The result of Figure 17 was also found in the single line fence case of Figure 9 and further suggests a deficiency in speed control loop of Figure 4.
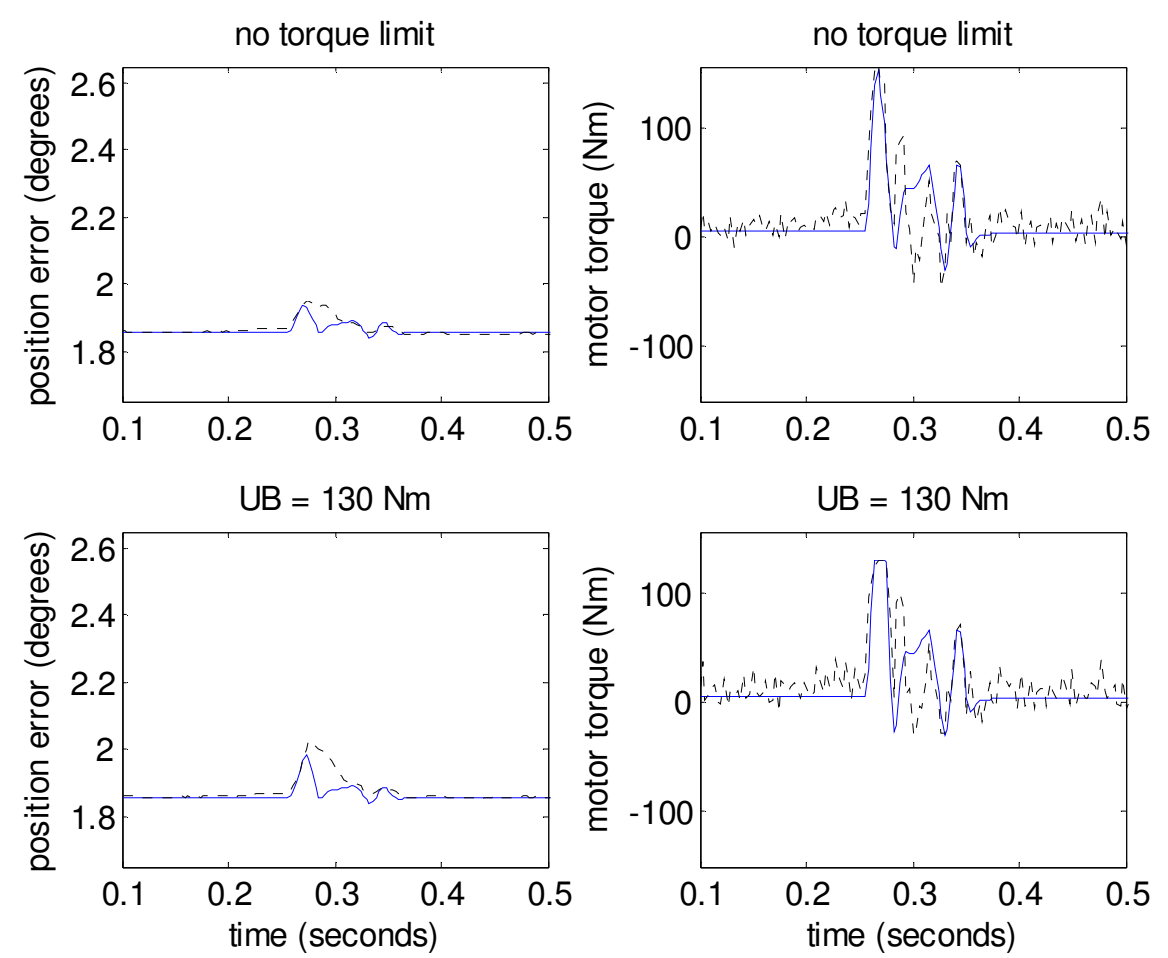

Figure 17: Reducing the proportional gain $\mathrm{K}_{2}$ from $30 \mathrm{~A}$ s/rad in Equation (6) to $8 \mathrm{~s} / \mathrm{rad}$

Also note that with no torque limit, the position error response is significantly more noisy during the period of maximum load, than with the torque limited responses of Figures 15-16. Figure 18 shows a close up of the no torque limit and three torque limited responses to show this effect in more detail. Figure 18 suggests there is an interaction between the control system and the main shaft that is not present in the torque limited responses. In the torque responses of Figures 15-16, there is a trend towards a constant torque during the maximum load, and thus effectively no control. In otherwords, it's the control system which is causing the extra noise in the no torque response of Figure 18, not the external load. This over response of torque from the control command and its effect on the position error provides more evidence of the deficiency in the speed control loop. 

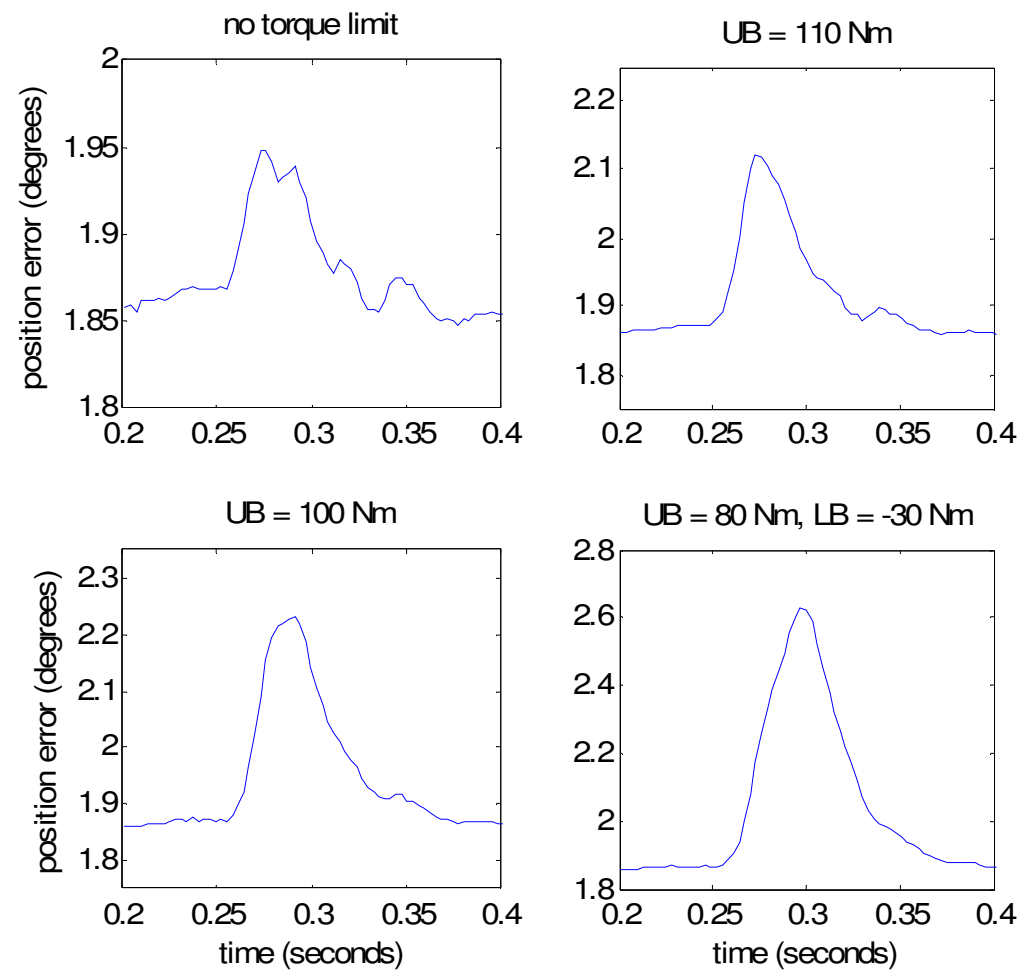

Figure 18: A close up of the position error responses during the load period. As the upper torque limit is reduced the response becomes more smooth.

This deficiency is likely due to some interaction between the commanded motor torque and the main shaft, for example torsional resonance effects from twisting in the shaft. This effect has been observed from the comparison of measured position at both ends of the shaft and the commanded motor torques.

A possible reason for the improved matches to the data in Figure 17 is that the twisting and other unknown effects are essentially lumped into the parameter $\mathrm{K}_{2}$. Hence even though $\mathrm{K}_{2}$ is physically set much higher in the actual machine, the net effect is a lower $K_{2}$ and less than optimal speed control loop. This result further shows the power of using this minimal modelling approach as various parameters can be tuned to capture non linear and complex effects relatively simply and with minimal computations.

As a final validation of the 6 data sets of Equation (25) a 6 fold validation is performed. That is, 5 of the data sets are used to predict a sixth data set for 6 combinations. The results of the model response are given in Table 2 where (1) refers to using the data sets $\{2, \ldots, 6\}$ to predict the data set(1), (2) refers to using the data sets $\{1,3, \ldots, 6\}$ to predict the dataset (2) 
and similarly for (3),..,(6). The errors in Table 2 are similar to Table 1 further validating the approach. The largest error is 0.096 in data set (6), but the position error dip in Figure 16 is correctly predicted and in percentage terms the error is $<5 \%$ of the offset position error (1.85 degrees) and is thus relatively small.

Table 2: Model response position error differences for main shaft using 5 data sets to predict a $6^{\text {th }}$ dataset in Equation (25)

\begin{tabular}{|l|l|l|l|}
\hline Fitted Data & Predicted Data & $\max \Delta \theta-\max \Delta \hat{\theta}$ & $\min \Delta \theta-\min \Delta \hat{\theta}$ \\
\hline$\{2,3,4,5,6\}$ & $(1)$ & 0.075 & -0.001 \\
\hline$\{1,3,4,5,6\}$ & $(2)$ & 0.082 & 0.004 \\
\hline$\{1,2,4,5,6\}$ & $(3)$ & 0.037 & 0.005 \\
\hline$\{1,2,3,5,6\}$ & $(4)$ & 0.013 & 0.018 \\
\hline$\{1,2,3,4,6\}$ & $(5)$ & 0.007 & 0.006 \\
\hline$\{1,2,3,4,5\}$ & $(6)$ & 0.001 & 0.096 \\
\hline
\end{tabular}

\subsubsection{Experimental Implementation of torque limits}

Overall, the measured and model responses of Figures 14-16 show that significantly less motor torque than the given measured motor torque in Figure 14 is required to adequately control the shaft position. In particular, only a change of 0.8 degrees is observed in Figure 16, with an upper torque limit of $80 \mathrm{Nm}$ as compared to the no torque limit in Figure 14. This change is well within the machine tolerances required for safety. Therefore, the maximum torque delivered by the motor could be made a lot less to minimize power consumption, and potentially, the motor could be downsized. However, note that in the machine, it was found that the $80 \mathrm{Nm}$ upper torque limit, was not sufficient to bring the main shaft up to speed.

Specifically, shortly after starting the machine, it immediately stopped due to the shaft position being too far away from the required reference position of Equation (1). This effect can also be observed in the model response. Figure 19 plots the model responses of the position error during the first revolution, for no applied torque limit versus the torque limit of $\mathrm{UB}=80 \mathrm{Nm}$ and $\mathrm{LB}=-30 \mathrm{Nm}$. 

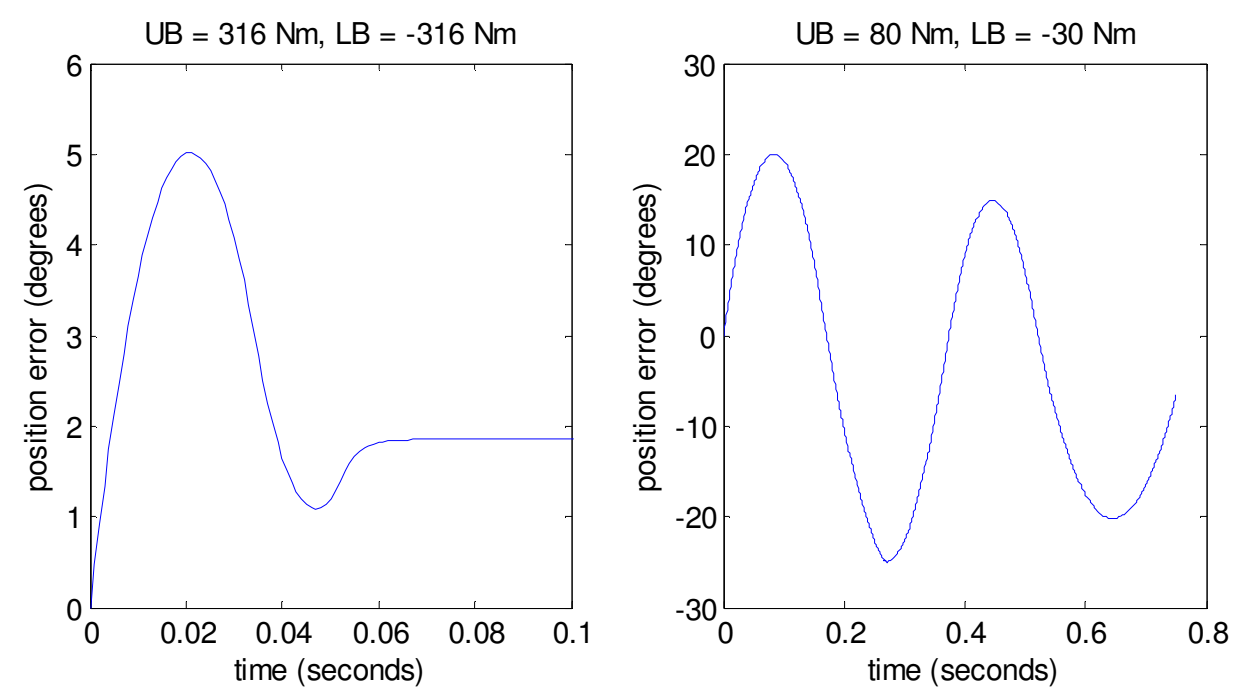

Figure 19: Reducing the proportional gain $\mathrm{K}_{2}$ from $30 \mathrm{~A}$ s/rad in Equation (6) to $8 \mathrm{~A}$ s/rad

In the first case, there is a natural torque limit of $\mathrm{UB}=316 \mathrm{Nm}$ and $\mathrm{LB}=-316 \mathrm{Nm}$ due to the physical constraints on the IFT6 synchronous motor as detailed in the configurational manual.

The no torque limited response in Figure 19 shows a very fast convergence to steady state after $\sim 0.06$ seconds. The maximum error is 5 degrees but occurs only briefly and is within machine tolerances. The $80 \mathrm{Nm}$ torque limited response has a position error still rising at $0.06 \mathrm{~s}$ which peaks at 20 degrees after $\sim 0.1 \mathrm{~s}$. This shows that the simulated main shaft position is falling dramatically behind the reference position, well outside machine tolerances. Even if the machine manages to get past the $20^{\circ}$ point, there is a swing in the negative direction of $46^{\circ}$ to $-26^{\circ}$ which would cause the machine tools to bang together, causing damage.

Therefore, in practise the machine needs to be run several times with a no torque limit to reach steady state, before a torque limit can be applied. This agreement between experiment and simulation further validates the model and approach and gives confidence in the accuracy of this simulation tool. 


\section{Conclusion}

The front main shaft and motor shaft control systems of a production machine were investigated for a single and dual line fence. Two different speeds and 6 combinations of motor torque were used. For the single line fence, several noise scenarios including time delay were considered to represent possible inaccuracies in the resolver for measuring position. The extra noise had a major effect on the net torque showing high sensitivity to position errors.

These results emphasise the importance of accurate position measurements, which could be achieved by for example an encoder. An encoder is known to be significantly more accurate than a resolver and is not sensitive to high accelerations which are present in this system. This behaviour has been seen experimentally with several experiments. An initial experiment involved the encoder on the end of the shaft (data not shown), which experiences greatly amplified effects of the torsional resonances. The resulting large accelerations would affect the accuracy of the feedback unit more than compared to placing it on the motor side which has lower accelerations.

Shifting the feedback from the far end of the shaft to the drive motor side greatly reduced the audible noise in the gearbox but not to an acceptable level. However, changing the integrated motor resolver to an encoder significantly reduced the audible noise, this time to an acceptable level. This trend for a smoother motor response as the accuracy in the position increases was observed in Figures 10-13 and provides another validation of the modelling approach in this paper.

The torque limiting experiments showed that significantly smaller motors were required to adequately control the main shaft position than are currently used on the machine. In particular, halving the maximum torque from $\sim 150 \mathrm{Nm}$ to $80 \mathrm{Nm}$ had less than 0.6 degrees changes in the position error. Smoother position responses were also observed during the period of maximum load as compared to the case of no torque limit. This observation demonstrates that the control system is certainly over responding and interacting with the shaft. 
The torque limiting responses also allowed an accurate identification of the external load on the machine. This method of torque limiting decouples the control system from the load, as during the main part of the load the applied torque is close to constant. The end result is an accurate method for identifying time varying loads on the machine. Future work could use this technique to correlate to different wire types and thus link wire types to engine requirements, which may help machine designs in the future.

Importantly, the mathematical model was rigorously validated and provides a means for testing many control scenarios without extensive time consuming testing and trial and error on the machine. The model and methods could be readily generalised to any other machine/control system of this type. 


\section{References}

[1] Renton D, Elbestawi MA. High speed servo control of multi-axis machine tools. International Journal of Machine Tools \& Manufacture. 2000;40(4):539-59.

[2] Elfizy AT, Bone GM, Elbestawi MA. Model-based controller design for machine tool direct feed drives. International Journal of Machine Tools \& Manufacture. 2004;44(5):465-77.

[3] Tao G, Kokotovic PV. Adaptive control of systems with unknown output backlash. IEEE Transactions on Automatic Control. 1995;40(2):326-30.

[4] Draca S. Finite element model of a double-stage helical gear reduction. Masters thesis, University of Windsor, Ontario, Canada http://13720714230/ web/vdc/downloads/library/theses/SDraca_mascpdf. 2006.

[5] Ozguven HN, Houser DR. Mathematical models used in gear dynamics - a review. Journal of Sound and Vibration. 1988;121(3):383-411.

[6] Singh R, Vinayak H. Multi-body dynamics and model analysis of compliant gear bodies. Journal of Sound and Vibration. 1998;210(2):171-214.

[7] http://www.automation.siemens.com/mc/mc-sol/en/f7642c37-0b26-48ff-ae00c0b0a548ed17/index.aspx.

[8] Hsue AWJ, Yan MT, Ke SH. Comparison on linear synchronous motors and conventional rotary motors driven Wire-EDM processes. Journal of Materials Processing Technology. 2007;192:478-85.

[9] Jing D, Jabbar MA, Zhejie L, editors. Dynamic Analysis of Interior Permanent Magnet Symchronous Machines. 2nd International Conference on Electrical and Computer Engineering ICECE; 2002; Dhaka, Bangladesh.

http://www.buet.ac.bd/eee/icece2002/paper_005.pdf.

[10] Van Haute S, Terorde G, Hameyer K, Belmans R, editors. Modelling and implementation of a permanent magnet synchronous motor drive using a DSP development environment. 8th International Power Electronics \& Motion Control conference (PEMC), 4:476-481; 1998; http://www.esat.kuleuven.be/electa/publications/fulltexts/pub_479.pdf.

[11] Rajanathan CB, Acikgoz H, Egin R. Transient characteristics of the single phase permanent magnet synchronous motor. IEEE Transactions on Magnetics. 1999;35(5):358991.

[12] Jia S, Howard I, Wang J. The Dynamic Modeling of Multiple Pairs of Spur Gears in Mesh, Including Friction and Geometrical Errors. International Journal of Rotating Machinery. 2003;9:437-42.

[13] Menon K, Krishnamurthy K. Control of low velocity friction and gear backlash in a machine tool feed drive system. Mechatronics. 1999;9(1):33-52.

[14] Odai M, Hori Y. Speed Control of Two-Inertia System with Gear Backlash Based on Gear Torque Compensation. Electrical Engineering in Japan. 2001;134(2):36-43. 
[15] Wang WJ, Chen JY. Passivity-based sliding mode position control for induction motor drives. Passivity-Based Sliding Mode Position Control for Induction Motor Drives. 2005;20(2):316-21.

[16] Encoder vs Resolver-Based Servo Systems. ORMEC Systems Corp http://wwwormeccom/LinkClickaspx?fileticket=I0Fu66PzelM\%3D\&tabid=91\&mid=866.

[17] Webster JG. The measurement, instrumentation and sensors handbook. Springer; 1999. 